PROCEEDINGS OF THE

AMERICAN MATHEMATICAL SOCIETY

Volume 137, Number 4, April 2009, Pages 1459-1465

S 0002-9939(08)09645-7

Article electronically published on October 29, 2008

\title{
BOUNDED HARMONIC 1-FORMS ON COMPLETE MANIFOLDS
}

\author{
M. COCOS
}

(Communicated by Varghese Mathai)

\begin{abstract}
In this paper we present some results concerning bounded harmonic 1-forms on manifolds of compact type. As a corollary we obtain a rigidity result for the first cohomology group of locally isometric Riemannian manifolds.
\end{abstract}

\section{INTRODUCTION AND STATEMENT OF MAIN RESULTS}

The classical De Rham-Hodge theory of harmonic forms on compact manifolds establishes a link between the underlying topology of the manifold and its geometric structure. The dimension of the spaces of harmonic forms is defined via the Riemannian structure and it is shown to be equal to the corresponding Betti number, which is a topological invariant. This is one of the first instances where topological quantities are obtained from geometric quantities. The first and the most celebrated example of this kind is the famous Gauss-Bonnet Theorem. In the case of noncompact manifolds little is known about the spaces of harmonic forms. It is most natural to ask whether in the noncompact case the dimensions of the spaces of harmonic forms are finite dimensional and also if they relate to the topology of the manifold. The study of the space of harmonic forms on noncompact manifolds may be used for the study of compact quotients of these manifolds as in Theorem 3.3. To be able to answer these questions one has to impose some additional conditions on the growth of the forms and/or the geometry of the manifold. For square-integrable forms classical results can be found in [10], [6, 7], 12, 1] and 8. In this paper we investigate the space of bounded harmonic 1-forms on certain manifolds. By the classical Hopf Theorem we know that on a compact manifold every harmonic function must be a constant. There are, however, numerous examples of noncompact manifolds which have the same property. A classical theorem of Yau states that on a complete manifold with nonnegative Ricci curvature there are no nonconstant, bounded harmonic functions. A more recent result is due to Avellaneda and Lin [2].

Theorem 1.1. Let the operator $L=\sum_{i, j=1}^{d}\left(\partial / \partial x_{i}\right)\left(a_{i, j}(x) \partial / \partial x_{j}\right), d \geq 1, x \in \mathbf{R}^{d}$, with periodic coefficients be such that $a_{i, j} \in \operatorname{Lip}\left(\mathbf{R}^{d}\right)$ and $\lambda|\xi|^{2} \leq \sum_{i, j=1}^{d} a_{i, j}(x) \xi_{i} \xi_{j}$ $\leq \lambda^{-1}|\xi|^{2}$ for all $\xi \in \mathbf{R}^{d}, x \in \mathbf{R}^{d}$, where the constant $\lambda \in(0,1]$. Then we have the following characterization of solutions of the equation:

Received by the editors February 28, 2008, and, in revised form, May 15, 2008.

2000 Mathematics Subject Classification. Primary 53C20.

(C)2008 American Mathematical Society Reverts to public domain 28 years from publication 
(1) $L u(x)=0\left(x \in \mathbf{R}^{d}\right)$ such that

(2) $\liminf _{R \rightarrow+\infty} R^{-\beta}\|u\|_{L^{\infty}(|x| \leq R)} \leq C$

for $\beta>0, C>0$ : Let $\nu_{n}=\left(\begin{array}{c}d+n-3 \\ n\end{array}\right)-\left(\begin{array}{c}d+n-1 \\ n-2\end{array}\right)$ for $n \geq 2, \nu_{0}=1, \nu_{1}=d$. There exists a sequence of linearly independent functions $\left\{Q_{n, j}(x) x \in \mathbf{R}^{d}, n \geq 0\right.$, $\left.1 \leq j \leq \nu_{n}\right\}$ satisfying $L Q_{n, j}(x)=0$ and $\left|Q_{n, j}(x)\right| \leq A_{n, j}|x|^{n}\left(A_{n, j}\right.$ independent of $x$ ), such that if $u$ satisfies (1) and (2), then $u(x)=\sum_{n=0}^{[\beta]} \sum_{j=1}^{\nu_{n}} c_{n, j} Q_{n, j}(x)$ for $c_{n, j} \in \mathbf{R}$ (the Liouville property).

If a metric on $\mathbb{R}^{n}$ is the pull-back of a metric from the $n$-dimensional torus $\mathbb{T}^{n}$ it will be called periodic. If one defines the action

$$
\mathbb{Z}^{n} \times \mathbb{R}^{n} \rightarrow \mathbb{R}^{n}
$$

in the standard way as

$$
\left(k_{1}, k_{2}, \ldots, k_{n}\right)\left(x_{1}, x_{2}, \ldots, x_{n}\right)=\left(x_{1}+k_{1}, x_{2}+k_{2}, \ldots, x_{n}+k_{n}\right),
$$

then an equivalent definition is that such a metric is invariant by the action defined in (1).

An immediate consequence of Theorem 1.1 is

Corollary 1.2. Let $g$ be a periodic metric on $\mathbb{R}^{n}$. If $f$ is a bounded harmonic function w.r.t. $g$, then $f$ is a constant.

These examples motivate the following definition:

Definition 1.3. Let $M$ be a complete Riemannian manifold. If on $M$ there is no nonconstant, bounded harmonic function, then $M$ is said to be of compact type.

Before we state our main result we must make a definition.

Definition 1.4. Let $\phi: M \rightarrow M$ be an isometry of the Riemannian manifold $M$. We say $\phi$ is bounded iff

$$
d_{M}(x, \phi(x))<C_{\phi} \quad \forall x \in M .
$$

Let $\beta_{1}(M)$ denote the dimension of the first De Rham cohomology group of the manifold $M$.

Our main result is:

Theorem 1.5. Let $M$ be a complete manifold of compact type with $\beta_{1}(M)=0$. If $\alpha$ is a closed, bounded and harmonic 1-form on $M$, then $\alpha$ is left invariant by any bounded isometry of $M$. In addition, if we assume that there is a group $G$ of bounded isometries which acts freely on $M$ such that the quotient $M / G$ is compact, then it follows that $\alpha$ is coclosed as well.

If $M$ is an $n$-dimensional Riemannian manifold, let $d$ denote the exterior derivative on $k$-forms and $*$ denote the Hodge isomorphism induced by the metric on $M$. Let $\delta=(-1)^{n k+n+1} * d *$ be the codifferential and $\Delta=d \delta+\delta d$ be the Laplace operators on forms. With this notation a form is closed iff $d \alpha=0$ and coclosed iff $\delta \alpha=0$. It is also very well known that on the flat Euclidean space $E^{n}$, all bounded harmonic forms are parallel and thus closed and coclosed. Here we have to point out the fact that in general even if we assume a harmonic form to be closed it is not true that it will be coclosed as well. For example $\alpha=x d x$ on $E^{2}$ is harmonic and closed but not coclosed, so it follows that even in the Euclidean case the boundedness of the form is essential. One other instance in which $\Delta \alpha=0$ implies $\left(d+d^{*}\right) \alpha=0$ is, 
for example, in our opinion, the fact that any sublinear growth harmonic function on a space with nonnegative Ricci curvature is constant (hence closed!). This is a consequence of Yau's gradient estimate [1] and it was proved by Cheng [4. The precise statement is:

Theorem (Cheng). Let $M$ be a complete Riemannian manifold with nonnegative Ricci curvature. Let $u$ be a sublinear growth harmonic function on $M$. Then $u$ must be identically constant.

It is therefore clear that even one of the most basic properties of harmonic forms on compact manifolds does not hold in the noncompact case. On the hyperbolic plane $\mathbb{H}^{2}$, for example, there are many bounded harmonic 1-forms which are not closed. To see this, one can consider

$$
\alpha=f(x, y) d x,
$$

where $f$ is the real part of a holomorphic function $F(z)$ satisfying

$$
|F(z)| \leq \frac{1}{|z|^{2}+1}
$$

Since on a 2-dimensional manifold any two metrics are locally conformal and the Laplacian in mid-degree is invariant to conformal transformations of the metric, it follows that $\alpha$ defined as in (2) is harmonic if and only if $f(x, y)$ is a harmonic function in the classical sense (i.e. is the real part of a holomorphic function). Moreover (3) will guarantee that $\alpha$ is bounded w.r.t. the hyperbolic metric. It is clear that only a subset of the set of all the forms defined as in (2) is closed.

The case of harmonic 1-forms is a very special one. By the classical Bochner argument one can see that on a compact manifold $M$ with positive Ricci curvature there is no nontrivial harmonic 1-form. Also on a compact manifold $M$, if the Ricci curvature is assumed to be nonnegative, a similar argument shows that the form has to be parallel. On a noncompact manifold with positive Ricci curvature, if a harmonic, bounded 1-form attains its maximum, the same arguments as in the compact case imply that the form is identically equal to zero. These arguments cannot be applied in the case when there is no assumption on Ricci curvature. A consequence of our main Theorem 1.5 is that bounded, closed and harmonic forms w.r.t. a periodic metric are pulled back from $\mathbb{T}^{n}$, and hence they form an $n$-dimensional vector space.

\section{Proof of the MAIN RESUlt}

We now give the proof of Theorem 1.5 .

Proof. Let $\alpha$ be the closed and bounded harmonic 1-form from the hypothesis and let $A>0$ such that

$$
\|\alpha\|_{L^{\infty}}<A
$$

Since $\beta_{1}(M)=0$ it follows that $\alpha$ is exact; hence

$$
\alpha=d f
$$

with $f$ a smooth scalar function defined on $M$. It follows that

$$
0=\Delta \alpha=\Delta d f=d \Delta f
$$


and consequently $\Delta f$ is constant. Let $\psi$ be a bounded isometry with respect to the metric $g$. This means that there exists a $C_{\psi}>0$ such that for every $x \in M$,

$$
d(\psi(x), x) \leq C_{\psi} .
$$

Next we have

$$
\Delta(f o \psi-f)=(\Delta f) o \psi-\Delta f=0 ;
$$

hence the function $h=f o \psi-f$ is harmonic w.r.t. $g$. We shall now prove that $h$ is bounded. Let us choose $c=c(t)$ to be a normalized geodesic joining $x$ and $\psi(x)$. The time interval of the geodesic is $0 \leq t \leq l$, and $l=d(\psi(x), x)$. We have

$$
|h(x)|=|f(c(l))-f(c(0))| \leq A d(\psi(x), x) \leq A C_{\psi} ;
$$

hence $h$ is bounded. Since $M$ has compact type it follows that $h$ is constant; therefore

or equivalently

$$
0=d(f o \psi-f)=\psi^{*}(d f)-d f,
$$

$$
\psi^{*}(\alpha)=\alpha .
$$

Let $G$ be as in the theorem and let $\theta$ denote the covering map

$$
\theta: M \rightarrow M / G \text {. }
$$

Since $\alpha$ is left invariant by all the elements of $G$, it follows that there exists $\beta$ a harmonic 1-form on $M / G$ such that

$$
\theta^{*}(\beta)=\alpha .
$$

Since $M / G$ is compact $\beta$ is coclosed and since $\delta$ commutes with local isometries, it follows that $\alpha$ is coclosed as well.

\section{A feW GeOMetric Applications of Theorem 1.5}

Corollary 3.1. Let $\mathbb{R}^{n}$ be the Euclidean space endowed with $g$ a periodic metric. Assume $\alpha$ is a closed, bounded, and harmonic 1-form with respect to $g$. Then $\alpha$ is left invariant by any isometry of $g$ which commutes with the action of $\mathbb{Z}^{n}$.

The proof of Theorem 3.1 in the case when $n=2$ is very simple. In this case since the metric is locally conformal to the Euclidean metric, it follows that the form is harmonic w.r.t. the Euclidean metric. Because the metric is periodic it is bounded w.r.t. the Euclidean metric and the form as well. Therefore the form has to be covariantly constant.

The proof for $n>2$ is a consequence of Theorem 1.5 and the following simple lemma:

Lemma 3.2. Let $M \mapsto N$ be a Riemannian cover with $N$ compact. Any isometry of $M$ which commutes with all the elements of the group of deck transformations of the cover is bounded.

Proof. Let $\psi$ be an isometry which commutes with all the elements of the group of deck transformations $G$. Let $D$ be a fundamental domain associated to the action of $G$ on $M$. Since $N$ is compact, it follows that $D$ is relatively compact. Take $x \in M$ to be an arbitrary point. Then there is $t \in G$ such that $t(x) \in D$ and since $\psi$ commutes with any element of $G$ it follows that

$$
d(x, \psi(x))=d(t(x), t(\psi(x)))=d(t(x), \psi(t(x))),
$$


and hence

$$
d(x, \psi(x)) \leq \sup _{y \in D} d(y, \psi(y)) .
$$

Since $D$ is relatively compact, $\sup _{y \in D} d(y, \psi(y))<\infty$ is attained and depends only on $\psi$.

Next we construct a natural (defined by the metric!) isomorphism between the first cohomology spaces of compact, orientable quotients of manifolds of compact type.

Corollary 3.3. Let $M$ be a simply connected, complete manifold of compact type. Let $M_{1}$ and $M_{2}$ be two orientable, compact manifolds covered by $M$. Assume that the groups of deck transformations associated to the two covers consist of bounded isometries. Then there is a natural isomorphism

$$
I: H_{D R}^{1}\left(M_{1}\right) \rightarrow H_{D R}^{1}\left(M_{2}\right)
$$

Proof. Let $[\alpha] \in H_{D R}^{1}\left(M_{1}\right)$ be a cohomology class. We want to define

$$
I([\alpha]) \in H_{D R}^{1}\left(M_{2}\right) .
$$

The covering map is a local diffeomorphism, and therefore $M_{1}$ and $M_{2}$ inherit natural metrics from $M$ which make the covering maps into local isometries. Since $M_{1}$ is compact and orientable, it follows that there is a unique $\left(d+d^{*}\right)$-harmonic form $\alpha \in[\alpha]$. If $\psi_{1}$ is the covering map

$$
\psi_{1}: M \rightarrow M_{1},
$$

then the pull-back

$$
\psi_{1}^{*}(\alpha)
$$

is a $\left(d+d^{*}\right)$-harmonic form on $M$. If $G_{2}$ denotes the group of isometries that generate $M_{2}$ in the sense that

$$
M_{2}=M / G_{2}
$$

then according to Theorem 1.5 it follows that $\psi_{1}^{*}(\alpha)$ is left invariant by $G_{2}$ and therefore there exists a unique harmonic form $\beta$ on $M_{2}$ such that

$$
\psi_{2}^{*}(\beta)=\psi_{1}^{*}(\alpha)
$$

Let us now define

$$
I([\alpha])=[\beta] .
$$

The map is well defined and linear. The inverse $I^{-1}$ is defined in an obvious way.

Remark. The conclusion of Corollary 3.3 stays the same if we assume that the two deck transformation groups commute.

The next result is concerned with the flow induced by the dual of a harmonic form.

Corollary 3.4. Let $M$ be a compact manifold. In addition assume that $M$ locally around any point has a frame of Killing vector fields. Then every bounded, closed, and harmonic 1-form is necessarily the dual of a Killing vector field. 
Proof. Let $\alpha$ be a form with the properties specified in the hypothesis. Let $p \in M$ and let $U$ be a neighborhood of the point $p$ where one can find a frame

$$
e_{1}, e_{2}, \ldots, e_{n}
$$

of Killing vector fields with associated coframe

$$
e^{1}, e^{2}, \ldots, e^{n}
$$

This means that the flow induced by these vector fields consists of isometries of the manifold. Now taking into account the conclusion of Theorem [1.5 we have

$$
\mathcal{L}_{e_{s}} \alpha=0 \text {. }
$$

By Cartan's formula

$$
\mathcal{L}_{e_{s}} \alpha=i_{e_{s}} d \alpha+d\left(i_{e_{s}} \alpha\right),
$$

and taking into account the fact that $\alpha$ is closed we conclude that

$$
\alpha\left(e_{s}\right)=a_{s},
$$

where $a_{s}$ is a constant real number. It follows that

$$
\alpha / U=\sum_{s=1}^{n} a_{s} e^{s}
$$

and the conclusion of the theorem follows.

\section{REFERENCES}

[1] Anderson, Michael T., $L^{2}$ harmonic forms on complete Riemannian manifolds. Geometry and analysis on manifolds (Katata/Kyoto, 1987), 1-19, Lecture Notes in Math., 1339, Springer, Berlin, 1988. MR961469 (89j:58004)

[2] Avellaneda, Marco; Lin, Fang-Hua, Une théorème de Liouville pour des équations elliptiques à coefficients périodiques (French. English summary). C. R. Acad. Sci. Paris Ser. I Math. 309 (1989), no. 5, 245-250. MR.1010728 (90j:35072)

[3] Carron, G., $L^{2}$-cohomology of manifolds with flat ends. Geom. Funct. Anal. 13 (2003), no. 2, 366-395. MR1982148 (2004e:53045)

[4] Cheng, S.Y., Eigenvalue comparison theorems and its geometric applications. Math. Z. 143 (1975), 289-297. MR0378001 (51:14170)

[5] De Rham, Georges, Differentiable manifolds. Forms, currents, harmonic forms. Grundlehren der Mathematischen Wissenschaften [Fundamental Principles of Mathematical Sciences], 266. Springer-Verlag, Berlin, 1984. MR760450 (85m:58005)

[6] Dodziuk, Jozef, Vanishing theorems for square-integrable harmonic forms. Proc. Indian Acad. Sci. Math. Sci. 90 (1981), no. 1, 21-27. MR653943 (83h:58006)

[7] Dodziuk, Jozef, $L^{2}$ harmonic forms on rotationally symmetric Riemannian manifolds. Proc. Amer. Math. Soc. 77 (1979), no. 3, 395-400. MR545603 (81e:58004)

[8] Gromov, M., Kähler hyperbolicity and $L_{2}$-Hodge theory, J. Differential Geom. 33 (1991), no. 1, 263-292. MR.1085144 (92a:58133)

[9] Li, Peter, Lecture notes on geometric analysis. Lecture Notes Series, 6. Seoul National University, Research Institute of Mathematics, Global Analysis Research Center, Seoul, 1993. MR1320504 (96m:58269)

[10] Vesentini, E., Lectures on Levi convexity of complex manifolds and cohomology vanishing theorems. Notes by M. S. Raghunathan. Tata Institute of Fundamental Research Lectures on Mathematics, No. 39, Tata Institute of Fundamental Research, Bombay, 1967. MR0232016 $(38: 342)$ 
[11] Yau, Shing Tung, Harmonic functions on complete Riemannian manifolds. Comm. Pure Appl. Math. 28 (1975), 201-228. MR0431040 (55:4042)

[12] Yau, Shing Tung, Some function-theoretic properties of complete Riemannian manifold and their applications to geometry. Indiana Univ. Math. J. 25 (1976), no. 7, 659-670. MR0417452 $(54: 5502)$

Department of Mathematics, Weber State University, 1702 University Circle, Ogden, Utah 84408-1702 